\title{
Role of ganglioside GM1 expression in T Iymphocytes membranes in cervical cancer development
}

\author{
Damir Danolić, Marija Heffer, Jasenka Wagner, Ivana Skrlec, Ilija Alvir, Ivica Mamić, Lucija Šušnjar, Luka \\ Marcelić, Tomislav Bečejac, Mario Puljiz
}

1 University Hospital Center Sestre Milosrdnice, University Hospital for Tumours, Zagreb, Croatia

${ }^{2}$ Faculty of Medicine, University of Osijek, Osijek, Croatia

${ }^{3}$ University Hospital Center Zagreb, Zagreb, Croatia

\section{Introduction}

Cervical cancer is worldwide the second most common cancer in females. Immune response appears to be a key determinant in controlling human papilloma virus (HPV) infection (1). Cell mediated immunity plays a significant role in progression or regression of neoplastic cervical lesions. Lipid rafts have essential role in T lymphocite activation $(2,3)$. Gangliosides play an important role in the formation and stabilization of specific cell lipid membrane domains $(4,5)$. This study aims to determine whether ganglioside GM1 expressions in $\mathrm{T}$ cell membrane represent risk factor for cervical cancer.

\section{Methodology}

In total, 20 women participated in our study. Peripheral blood samples were obtained from 10 cervical cancer patients and 10 healthy controls at University Hospital for Tumors, Zagreb, Croatia. A $3 \mathrm{~mL}$ sample of venous blood was collected from each subject into a test tube containing EDTA as anticoagulant. For $T$ cell subtypes isolation we used Anti CD4 Phycoerythrin antibody and Anti CD8 Peridinin Chlorophyll Protein Complex antibody. Ganglioside GM1 expression in T lymphocytes membranes was analyzed using double labeling antibodies on ganglioside GM1 and cholera toxin.

\section{Results}

We have not found difference in ganglioside GM1 expression in $\mathrm{T}$ lymphocytes membranes between the two groups (Figures 1 . and 2.).

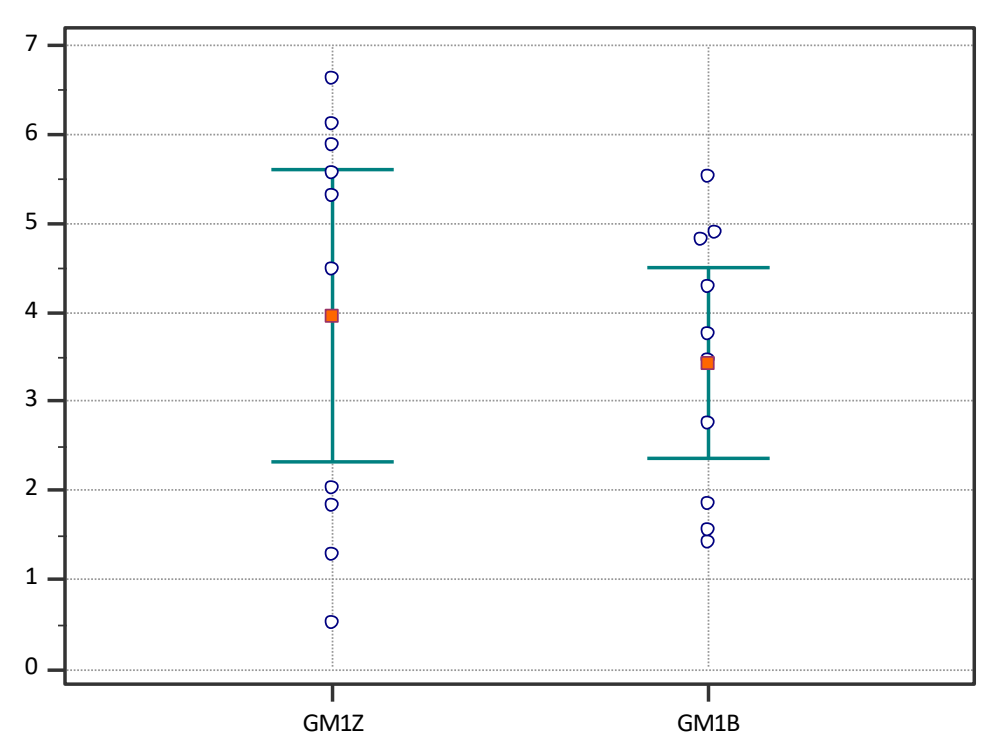

Figure 1. Comparison of ganglioside GM1 expression in T lymphocytes membranes using double labeling antibodies on ganglioside GM1. The difference between the groups was not statistically significant $(t=-0.67$; $d f=18 ; P=0.549$ ).

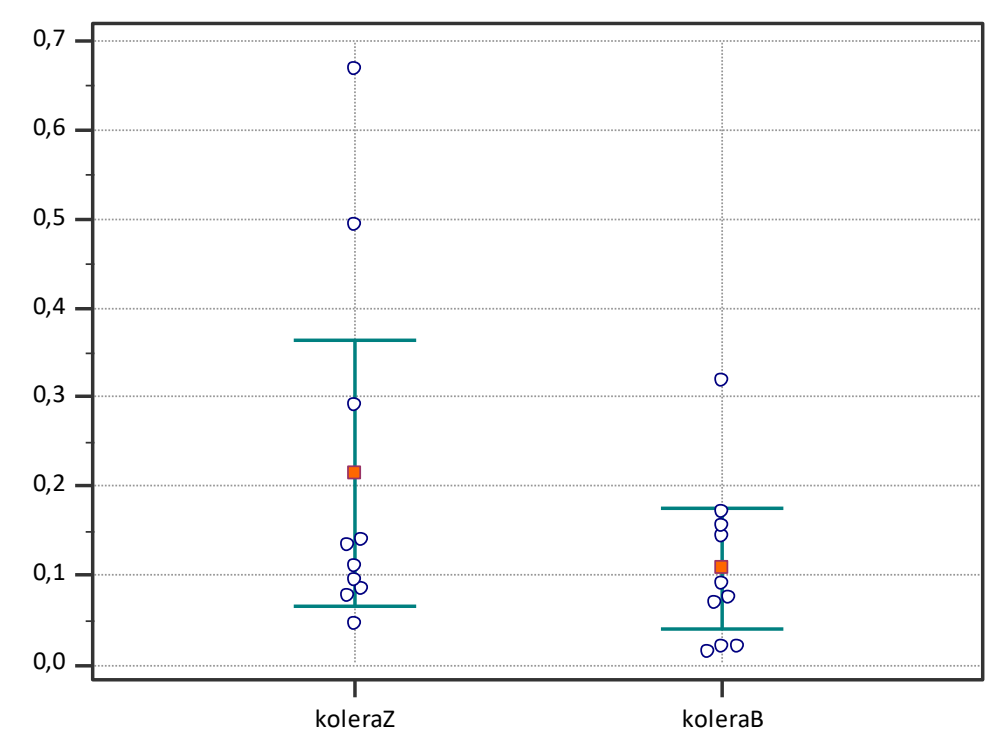

Figure 2. Comparison of ganglioside GM1 expression in T lymphocytes membranes using cholera toxin. The difference between the groups was not statistically significant $(t=-1,468 ; d f=18 ; P=0,166)$.

\section{Conclusion}

The present study did not show association of ganglioside GM1 expression in $\mathrm{T}$ lymphocytes membranes with cervical cancer development.

References

1. Stanley M. Immune responses to human papillomavirus. Vaccine 2006 30;24 (Suppl 1): S16-22.

2. Inokuchi J, Nagafuku M, Ohno I, Suzuki A. Distinct selectivity of gangliosides required for CD4+T and CD8+ T cell activation. Biochim Biophys Acta. 2015; 1851: 98-106.

3. He HT, Lelloucha A, Margueta D. Lipid rafts and the initiation of T cell receptor signaling. Seminars in Immunology. 2005; 17: 23-33.

4.Sonnino S, Mauri L, Chigorno V, Prinetti A. Gangliosides as components of lipid membrane domains. Glycobiology. 2007 ; 17 : 1-13.

5..Lopez PH, Schnaar RL. Gangliosides in cell recognition and membrane protein regulation. Curr Opin Struct Biol. 2009; 19 : 549-557.
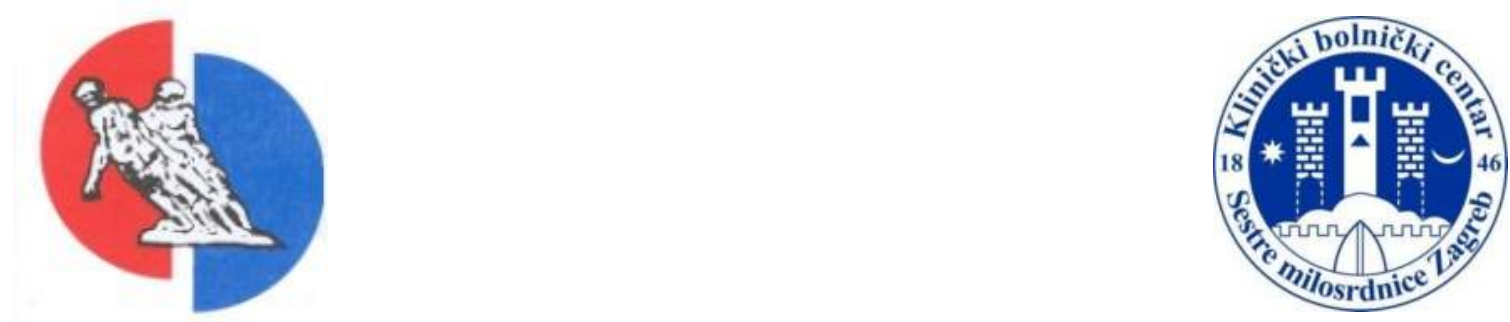